\title{
Use of a Second Ray Amputation for Foot Salvage in a Collegiate Athlete with Proteus Syndrome
}

\author{
Ethan R Harlow, $M D^{1}$, Pranav Khambete, $B S^{2 *}$, Jason Ina, $M D^{1}$, and Shana Miskovsky, $M D^{1,3}$ \\ ${ }^{1}$ Department of Orthopaedic Surgery, University Hospitals Cleveland Medical Center, USA \\ ${ }^{2}$ Case Western Reserve School of Medicine, Cleveland OH, USA \\ ${ }^{3}$ Sports Medicine Institute, University Hospitals Cleveland Medical Center, USA
}

*Corresponding author: Pranav Khambete, Case Western Reserve School of Medicine, Cleveland OH, USA

\section{Introduction}

Proteus syndrome (PS) is a rare disease of asymmetrical hamartomatous overgrowth only recently delineated in 1979 and named in 1983 for a Greek god that was reputed to be polymorphous [1-3]. It is known to affect multiple tissues, predominantly connective tissue and bone $[3,4]$. Proteus syndrome is characterized by the presence of mosaic, progressive, and sporadic overgrowth lesions. These often occur asymmetrically in the distal limbs, but can affect any part of the body. The exact etiology of PS has not been elucidated, but it is likely due to a somatic mutation distributed in a mosaic fashion, accounting for the wide variability of lesions observed between patients [2,4]. Numerous criteria were established in 1998 by the NIH to diagnose PS, notably disproportionate limb overgrowth causing loss of function of the affected limbs [5]. In this report, we describe a rare case of PS in a collegiate soccer player presenting with right foot pain and asymmetric overgrowth. In review of the literature, there are no reported cases of patients with disproportionate limb overgrowth attributed to PS that are able to compete athletically at this level. A report is available of PS in a young male with bilateral megafoot who was able to play soccer through adolescence; however, this patient did not report pain, functional limitations, or seek treatment [6]. Moreover, there is little literature guiding clinicians on whether or not high-level activity can be preserved through limb salvage procedures in populations with congenital malformations and oncological disorders of the forefoot. This case describes a second ray amputation performed for severe forefoot deformity with the goal of salvaging the foot, restoring high-level function, and improving the cosmetic appearance. This utilitarian surgical technique can also be applied in the treatment of patients with challenging deformities, infections, traumatic injuries and tumors.

\section{Case Description}

\section{Patient presentation}

Our patient is a 20-year-old female, NCAA Division II collegiate soccer player, who presented to the office with progressive deformity and pain of the right foot and second toe that has been worsening over one year. At age 5, she was diagnosed with PS with the right foot overgrowth as the presenting symptom. Her symptoms progressed to the point where prolonged standing would cause her pain and thus rendered her unable to continue her athletic endeavors, limited her shoe wear, and began to affect her ability to perform every day activities. Due to the deformities and multiple bony masses, she was forced to wear a size 11 shoe on the right foot, as compared to size 7 on the uninvolved, left foot. On exam, she has developed thick callosities in the plantar webspace in between the first and second metatarsal and plantar-medial aspect of the great toe. Skin changes typical of PS, cerebriform lesions, were present on the plantar-medial aspect of her right foot (Figure $1 \mathrm{~A}$ and Figure 1B). Neurologic exam was normal. Vascular exam revealed 2+ pedal pulses and markedly enlarged veins were observed

Citation: Harlow ER, Khambete P, Ina J, Miskovsky S (2021) Use of a Second Ray Amputation for Foot Salvage in a Collegiate Athlete with Proteus Syndrome. Int J Foot Ankle 5:052. doi.org/10.23937/26433885/1710052

Accepted: February 20, 2021; Published: February 22, 2021

Copyright: (C) 2021 Harlow ER, et al. This is an open-access article distributed under the terms of the Creative Commons Attribution License, which permits unrestricted use, distribution, and reproduction in any medium, provided the original author and source are credited. 

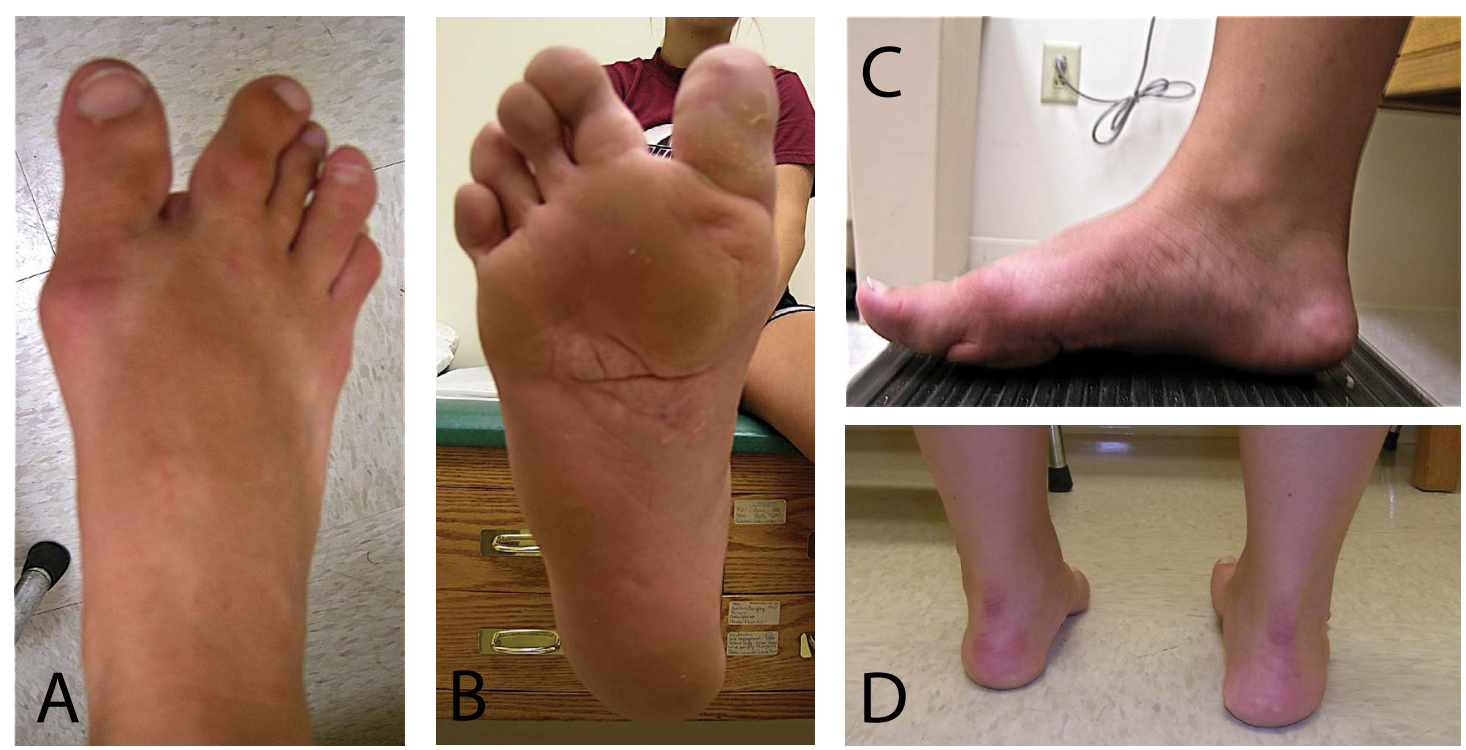

Figure 1: Right foot with typical morphological appearance associated with Proteus syndrome, including secondary skin and foot deformities due to heterotopic ossification and plantar skin cerebriform lesion: (A) Dorsal view, (B) Plantar view, and (C) Medial weightbearing side view. Second toe overgrowth and formation of large heterotopic ossification in the first webspace lead to first ray elevation off of the ground, hindfoot varus posture, and subsequent lateral overload syndrome (D).
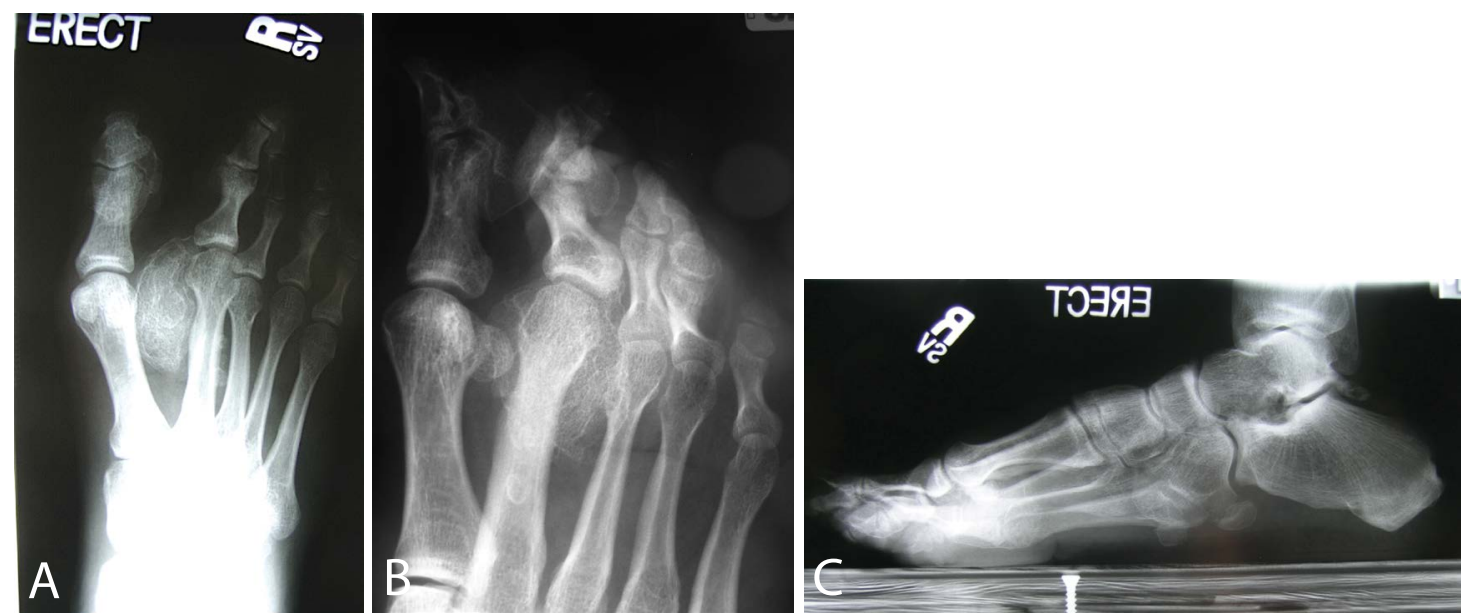

Figure 2: Weightbearing X-ray films of the right foot revealed a large amount of well-corticated heterotopic ossification encompassing and encircling the second metatarsal, along with smaller bony masses over the plantar-medial aspect of the great toe, plantar PIP area of the third toe and lateral border of foot: (A) AP view, (B) Oblique view and (C) Lateral view.

in both extremities. Limited motion of the metatarsophalangeal and interphalangeal joints of all digits was noted for the right foot. The second toe had significant overgrowth and an associated bony prominence between the first and second metatarsals measuring $3.5 \times 3 \mathrm{~cm}$. Upon weight bearing, this would push her great toe cephalad causing the toe to not sit properly on the ground, creating a dynamic cock-up toe deformity. The hindfoot was in varus with weight bearing, along with her great toe not making appropriate ground contact. A cavovarus foot posture resulted with development of a painful lateral overload syndrome (Figure 1C and Figure 1D). Weight-bearing $\mathrm{X}$-rays demonstrated a large amount of well-corticated heterotopic bone formation in the first webspace encircling the distal neck and head region of the right second metatarsal. Other bony masses were identified at the plantar-medial aspect of the great toe,
Plantar Proximal Interphalangeal (PIP) of the third toe and, to a lesser extent, lateral border of the foot (Figure $2 \mathrm{~A}$, Figure $2 \mathrm{~B}$ and Figure $2 \mathrm{C}$ ).

\section{Discussion of treatment}

Treatment of the above foot deformity with heterotopic ossifications is not straightforward. Initially, due to the patient's hopes of continuing to play soccer and due to her intense class schedule, the patient elected to use custom full length total contact inserts with relief channels to offload bony prominences and shoe wear modifications including high top type of shoes or men's shoes. She was able to return to her soccer participation for a period of time with cleat adjustments and multiple pairs of orthotic inserts. However, three years later, her symptoms recurred and prevented her from competing at her desired level and pain was progressing 
even with daily activities or light exercise. At this point, the discussion of surgical treatment took place including the following options: First webspace heterotopic bone removal only, second ray amputation, transmetatarsal amputation (due to other lesions present), or below knee amputation. It was evident that heterotopic ossification was encasing the neurovascular bundles supplying the second toe, making an isolated mass excision likely to render the second toe dysvascular or non-functional, ultimately necessitating future amputation. After a thorough review of the advantages and disadvantages of each surgical intervention, second ray amputation with removal of the accompanying heterotopic ossification was chosen to achieve the goal of functional preservation and creation of a more shoeable and plantigrade foot. Due to the large area of heterotopic ossification, the patient was instructed that surgical procedures would be staged to minimize chance of neurovascular injury and wound healing complications.

In review of the literature, such a procedure has been described in a case series by Turra, et al. where a ray amputation was indicated in a case of severe foot enlargement, multiple failed procedures, and an insensate toe [7]. However, when to pursue a corrective surgery in PS
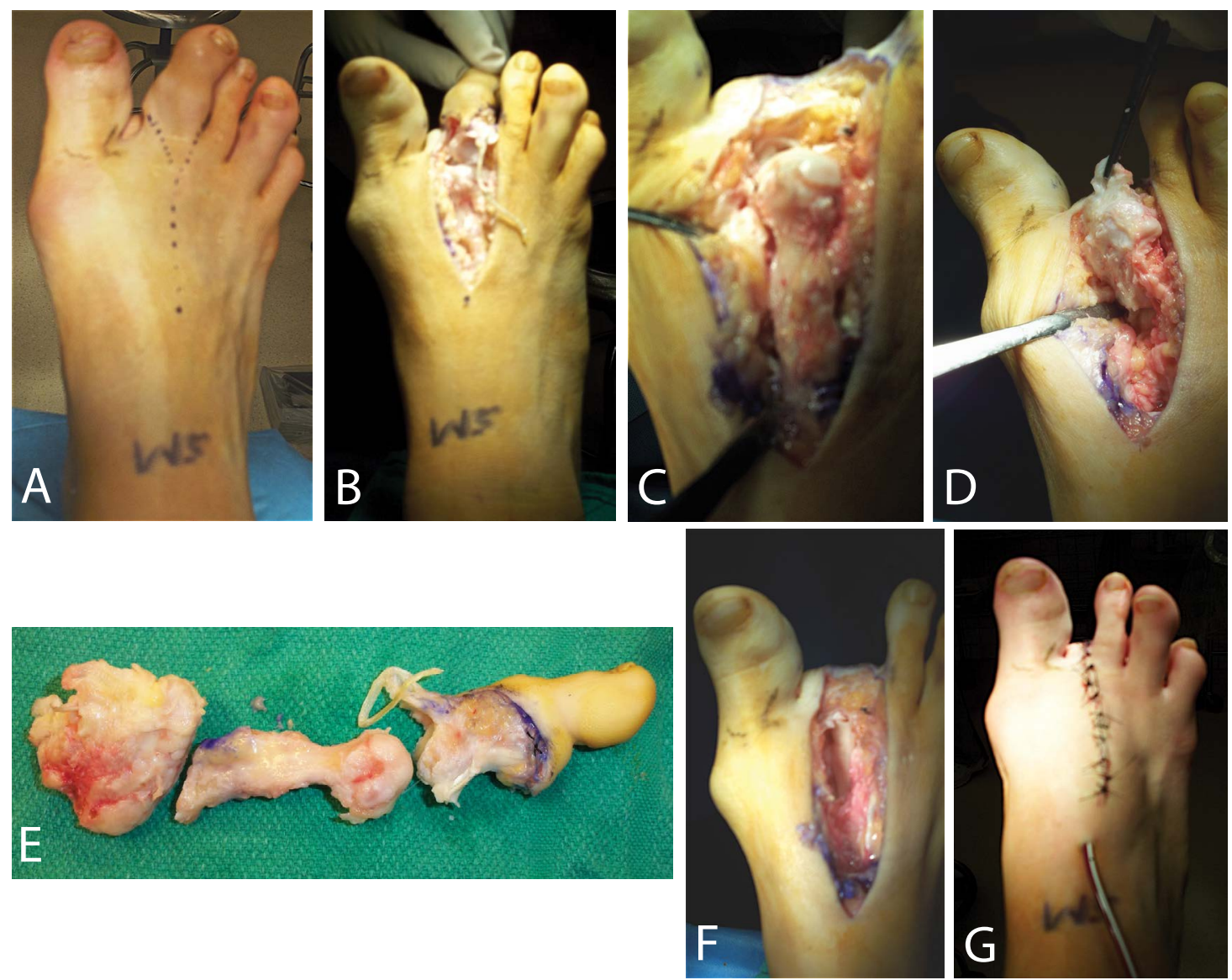

Figure 3: Illustrations of surgical technique for second ray amputation and removal of large heterotopic ossification in first webspace: (A) Racquet-shaped surgical incision outlined, (B) Neurovascular bundles to the second toe identified with artery ligated and digital nerve sharply transected, (C) Level of second metatarsal osteotomy identified, (D) Second metatarsal osteotomy completed in the proper orientation with heterotopic bone mass seen, $(E)$ Second toe specimen prepared and sent to pathology, and (F) Residual space in between the first and third metatarsal shafts then (G) Reduced utilizing nonabsorbable suture passage around first and third metatarsals with placement of deep drain and skin closure. 


\section{Second ray amputation surgical technique}

Patient was positioned supine with a bump under the right hip and a tourniquet was insufflated. With a skin marker, a dorsal longitudinal incision following the shaft of the second metatarsal and then curving around the base of the $2^{\text {nd }}$ toe at the level of the metatarsophalangeal joint (racquet-type incision) was planned (Figure $3 \mathrm{~A})$. Incision was performed with 15-blade and hemostasis was maintained utilizing bipolar cautery. Loupe magnification was used to aid in visualization of neurovascular bundles and deep structures during surgery. Incision was completed and neurovascular bundles to the second toe were isolated (artery tied off with 3-0 silk and digital nerve sharply transected) (Figure 3B). The capsule of the $2^{\text {nd }}$ MTP joint, extensor tendons and flexor tendons were cut with tension applied and the toe was removed, leaving the second metatarsal. The dorsalis pedis and first intermetatarsal artery were seen and protected. The osteotomy cut was localized to the base of the second metatarsal approximately $2 \mathrm{~cm}$ distal to the $2^{\text {nd }}$ tarsometatarsal joint (Figure $3 \mathrm{C}$ ) and confirmed with fluoroscopy. Small hohmann retractors were placed underneath the second metatarsal shaft at the site of the osteotomy cut and rotated so as to avoid contact with the saw and to protect the surrounding soft tissues. Osteotomy of the $2^{\text {nd }}$ metatarsal base was performed with an oscillating saw oriented perpendicular to the shaft of the metatarsal aiming in a dorsal-distal to plantar-proximal orientation to avoid a bony prominence on the plantar aspect of the foot (Figure 3D). A thin, straight osteotome was utilized to help mobilize the osteotomy. A Kocher clamp was placed on the $2^{\text {nd }}$ metatarsal and a beaver blade was used to free the soft tissues from the second metatarsal and osteotomes were used to release portions of the bony "noose" around the second metatarsal. Once free, the second metatarsal was removed, exposing the large extent of the heterotopic bone formation in the first webspace. After the bony overgrowth was carefully dissected and removed, the second toe specimen was prepared (Figure 3E) and sent to the pathologist who later confirmed the mass to be heterotopic bone formation. To aid in maintaining the new corrected width of the foot and to decrease tension on the dorsal wound (Figure 3F), a 90 degree clamp was utilized to free the soft tissues about the shaft of the first metatarsal and third metatarsal. Using the clamp, a number 2 FiberWire (Arthrex, Inc, Naples, Florida) was passed around the first and third metatarsal shafts respectively and, with a compression force applied to the remaining metatarsals in the coronal plane, the suture was tied and secured. After copious irrigation, a 7 french drain attached to a hemovac was placed in the wound and wound closure was performed utilizing mattress sutures with 3-0 nylon to keep as little tension on the wound as possible (Figure 3G). Gentle skin handling was utilized. Dressing was placed and tourniquet was released with all toes pink with good capillary refill con- firmed prior to sterile drapes removed. A well-padded short leg bivalved cast was applied to the right lower leg.

\section{Surgical course and outcome}

Radiation therapy with 700 cGy was administered to the right foot in one treatment session was performed following surgery in an attempt to prevent regrowth of the heterotopic bone. Post-operatively, she was placed in a short leg non-weight bearing cast until wound healing occurred uneventfully and the sutures were removed. Then, the second, staged surgery was performed on 21 days later during which several corrective measures were undertaken: 1 ) removal of heterotopic bone mass from the plantar aspect of the great toe which was causing essentially a cock up Deformity; 2) Correction of great toe deformity (lengthening the extensor hallucis longus and brevis tendons, releasing the tight dorsal capsular structures and percutaneous pinning); 3) Removal of heterotopic bone from plantar aspect of $3^{\text {rd }}$ toe PIP joint area; and 4) Correction of $4^{\text {th }}$ toe deformity (via extensor tendon lengthening, dorsal capsulotomy and percutaneous pinning). The patient was kept non-weight bearing until pins were removed four weeks post-surgery. She went on to resume her college classes and was able to resume a vigorous walking program, but not return to competitive soccer. One year later, the patient developed dorsal great toe metatarsophalangeal discomfort related to her hallux rigidus which progressively limited her daily walking activities. Surgery was performed 1 year later in the form of a right great toe cheilectomy and capsular release with removal of the medial eminence and the dorsal one-third of the metatarsal head. Four years after her second ray amputation, she is progressing well, graduating college, and participates regularly in exercise walking and other low-impact athletic activities. She was satisfied with her result. There is no evidence of re-growth of masses in the right foot. She is now able to wear a size 8 shoe on the right foot. The clinical appearance of her right foot is shown in (Figure 4).

\section{Conclusions}

Patients with limb overgrowth resulting in significant forefoot deformity are difficult to manage and this case demonstrates the utility of a second ray amputation in salvaging the foot, restoring adequate function, and improving cosmetic appearance in a patient with Proteus syndrome. A detailed knowledge of both conservative and surgical treatment options can aid the surgeon in determining the most appropriate management rationale in light of the patient's age, health, activity level, functional limitations and outcome expectations. Second ray amputation may be considered as a foot salvage option for certain patients with challenging deformities, infections, traumatic injuries and tumors. Utilizing this approach with careful attention to surgical technique as described, more proximal limb amputations can hope- 

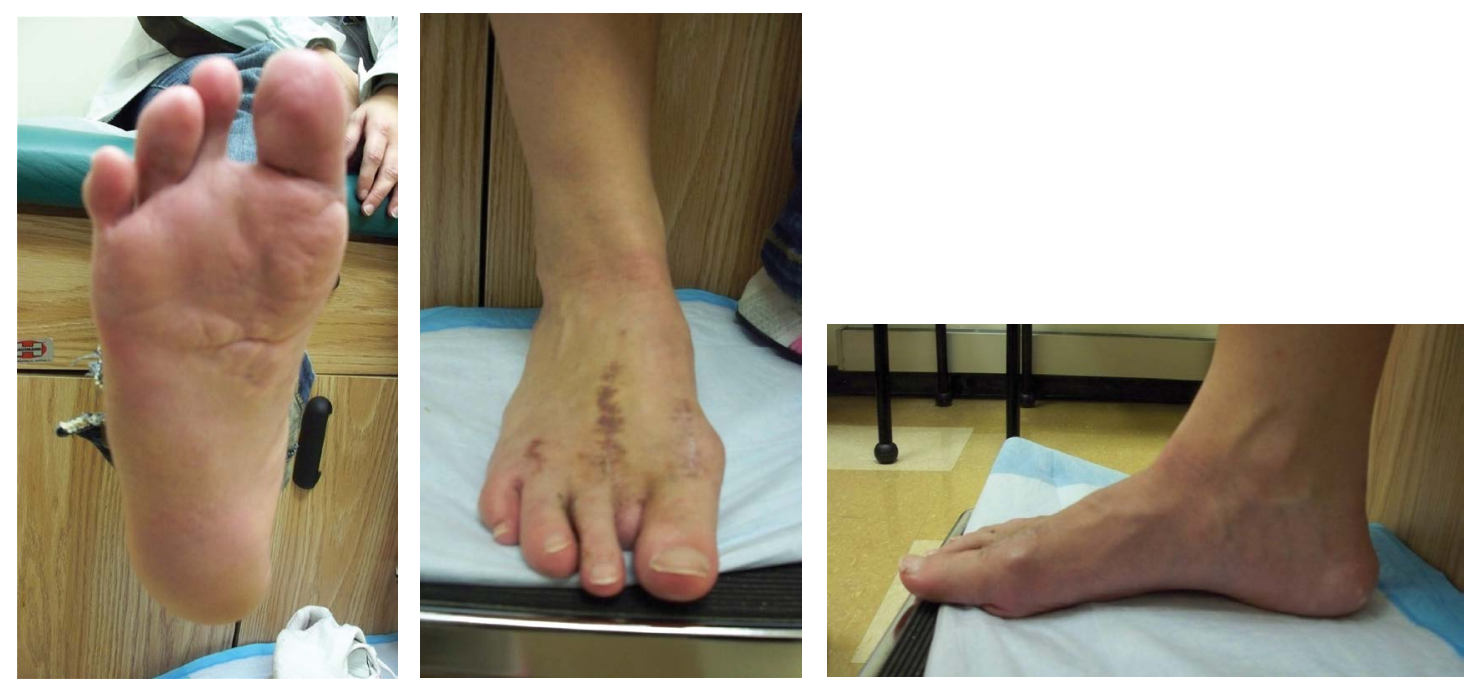

Figure 4: Clinical appearance of right foot after second ray amputation and other minor procedures, allowing the patient to wear a size 8 shoe.

fully be avoided; thus minimizing concerns with respect to long term prosthetic use, residual $\operatorname{limb}$ breakdown, and increased energy expenditures with activity.

\section{Authors Declarations}

The authors above have no financial disclosures or conflicts of interest.

\section{Statement of Authors' Contributions}

ERH: Drafted the manuscript; PK and JI: Contributors in the writing and editing of the manuscript; SM: Supervisor, oversaw treatment of the patient, drafted and critically revised manuscript; All authors read and approved the final manuscript.

\section{References}

1. Cohen Jr MM (2005) Proteus syndrome: An update. Am J Med Genet C Semin Med Genet 137C: 38-52.

2. Hoeger PH, Martinez A, Maerker J, Harper JI (2004) Vascular anomalies in proteus syndrome. Clin Exp Dermatol 29: 222-230.

3. Weidemann HR, Burgio GR, Aldenhoff $P$, J Kunze,
Kaufmann HJ, et al. (1983) The proteus syndrome: Partial gigantism of the hands and/or feel, nevi, hemihypertrophy, subcutaneous tumors, macrocephaly or other skull anomalies and possible accelerated growth and visceral affections. Eur J Pediatr 140: 5-12.

4. Biesecker $L$ (2006) The challenges of proteus syndrome: Diagnosis and management. Eur J Hum Genet 14: 11511157.

5. Biesecker LG, Happle R, Mulliken JB, Weksberg R, Graham Jr JM, et al. (1999) Proteus syndrome: Diagnostic criteria, differential diagnosis, and patient evaluation. Am J Med Genet 84: 389-395.

6. Derman O, Balci S, Engiz O (2008) Approach in an adolescent proteus male patient with megafoot. Turk J Pediatr 50 : 200-203.

7. Turra S, Santini S, Cagnoni G, Jacopetti T (1998) Gigantism of the foot: Our experience in seven cases. J Pediatr Orthop 18: 337-345.

8. Ozturk H, Karnak I, Sakarya MT, Cetinkursun S (2000) Proteus syndrome: Clinical and surgical aspects. Ann Genet 43: $137-142$.

9. Linton JA, Seo BK, Oh CS (2002) Proteus syndrome: A natural clinical course of proteus syndrome. Yonsei Med J 43: 259-266. 